\title{
LA GESTIÓN DEL RIESGO BAJO UN ENFOQUE SOSTENIBLE Y DE DERECHOS*
}

Artículo de Investigación - Recibido: 03 de octubre de 2014. Aceptado: 12 de enero de 2015

\author{
Carmen Meza* \\ Universidad de la Costa CUC, Barraquilla - cmeza2@cuc.edu.co
}

\section{Para citar este artículo / to reference this article:}

Meza, C. (2015). La gestión del riesgo bajo un enfoque sostenible y de derechos. Módulo Arquitectura CUC, Vol.14 №2 41-62. Doi:

\section{Resumen}

El riesgo está implícito en cada dinámica de ocupación, producción y transformación que impone el ser humano en los territorios; el enfoque de derechos implica aumentar las competencias y capacidades de acción de las personas, organizaciones y sociedades para tomar decisiones asociadas al desarrollo. Promover la sostenibilidad ambiental, social y económica debe ser el principio que guíe la formulación de las políticas públicas locales para la gestión de un plan de ordenamiento territorial que logre por medio de acciones estratégicas disminuir los factores de riesgo. El presente artículo es el resultado de una investigación elaborada en el marco de los proyectos de desarrollo y paz en la Región Caribe, una apuesta a la construcción de escenarios colectivos de participación para la construcción de estrategias de fortalecimiento institucional, rol que desempeñan las organizaciones públicas y privadas para el manejo de la gestión del riesgo en cada municipio.

\section{Palabras claves:}

Gestión del riesgo, ordenamiento territorial, políticas públicas, desarrollo y vulnerabilidad.

\footnotetext{
* Artículo resultado de la investigación elaborada en el marco de los proyectos de desarrollo y paz en la Región Caribe, elaborada por bajo el Grupo de investigación ARUCO, Línea de Investigación Calidad del Hábitat y el Entorno.

** Arquitecta. Magister en Planificación Territorial y Desarrollo Regional Universidad de Barcelona. Candidata a Doctora en geografía, planificación territorial y gestión ambiental de la Universidad de Barcelona. Docente de la facultad de arquitectura de la Universidad de la Costa-CUC.
} 


\section{LOW RISK MANAGEMENT APPROACH TO SUSTAINABLE AND RIGHTS}

\section{Abstract}

The risk is implicit in each occupation dynamics, production and processing imposing man in the territories, the rights approach involves increasing the skills and capacity for action of individuals, organizations and societies to make decisions related to development. Promoting environmental sustainability, social and economic should be the guiding principle in the formulation of local policies for managing land use plan that achieved through strategic actions reduce risk factors. This article is the result of a consultancy developed in the framework of peace and development projects in the Caribbean region, a commitment to building collective participation scenarios for building institutional strengthening strategies, role played by public organizations and private management of risk management in each municipality.

\section{Key Words}

Risk management, land management, public policy, development and vulnerability. 


\section{Introducción}

La gestión del riesgo como ámbito de política pública debe contribuir al desarrollo seguro y sostenible. La Conferencia Internacional sobre Reducción de Desastres Naturales: "Por un Mundo Más Seguro en el Siglo XXI", celebrada en Jokohama, Japón, en 1994, destacó los vínculos entre la reducción de desastres y el desarrollo sostenible. Bajo este razonamiento, enmarca el problema desde el contexto ambiental y reconoce la relevancia de la prevención como forma de reducir los desastres. Según el Plan Nacional para la Gestión del Riesgo 2010-2015 (PNGR) de Costa Rica, la gestión del riesgo debe mantener la perspectiva analítica potenciando la crítica y el análisis de los factores de riesgo dentro la dimensión totalizadora de la sostenibilidad ambiental, teniendo presente que los factores de riesgo inherentes al desarrollo amenazan la seguridad y el bienestar humano.

El enfoque de derechos implica aumentar las competencias y capacidades de acción de las personas, organizaciones y sociedades para tomar decisiones asociadas al desarrollo. Estas decisiones deben permitir solucionar sus problemas y redundar en el control y disminución de los factores de riesgo, así como de la protección de la integridad física y la salvaguarda de los bienes materiales y de la vida misma.

La debilidad institucional y el desconocimiento de los derechos fundamentales así como la baja o nula participación ciudadana en términos de asociatividad, aumentan los niveles de riesgo impidiendo respuestas rápidas y efectivas ante situaciones adversas; la propuesta que se presenta plasma el análisis de la gestión de riesgo de manera articulada a los distintos enfoques de intervención en el marco de las dimensiones del desarrollo.

El artículo es el resultado del análisis de información de base relacionada con el tema de la gestión de riesgo y las dimensiones del desarrollo desde un enfoque territorial, así como, la inclusión de los resultados obtenidos en talleres participativos llevados a cabo en varios municipios del departamento del Atlántico, con el objetivo de identificar el nivel de conocimiento de las comunidades sobre el tema del riesgo y de derechos asociado a la gestión y participación de los mismos, en la formulación de las políticas públicas para la intervención en los modelos de planificación y ordenamiento territorial que por ley deben incorporarse. El proyecto tiene como objetivo principal el diseño de una propuesta metodológica que oriente la inclusión del enfoque de derechos al concepto de gestión del riesgo de manera transversal a los distintos enfoques de intervención territorial.

El contenido general desarrolla tres partes: el primero, es el marco referencial del documento el cual aborda el tema de la gestión del riesgo desde los marcos 
normativos, los referentes nacionales y las estructuras institucionales, se considera necesario explicitarlo para entender el marco de política en el cual se deben desarrollar y amparar las estrategias de acción que se propongan y como la cooperación internacional juega un rol importante en el acompañamiento de la financiación de los emprendimientos que se gesten como resultado de la gestión municipal.

La segunda parte da cuenta de la metodología utilizada y los resultados obtenidos de la información de campo, explicando las dinámicas, problemas y dificultades presentes que impiden el funcionamiento y relacionamiento de las organizaciones sociales con el resto de actores municipales; este es el insumo para poder avanzar en el planteamiento de acciones concretas de fortalecimiento, trabajo interinstitucional y de cooperación.

Finalmente, se describe la estrategia cuyo objetivo principal es dar las pautas sobre cómo debe articularse la institucionalidad pública con los entes privados y de cooperación para llevar a cabo acciones concretas sobre el manejo de la gestión de riesgo y su incorporación en los planes de ordenamiento territorial , no solo como una definición de lo físico si no como política pública bajo un enfoque de derechos, para la gestión y conformación de los grupos de acción que garanticen la inversión pública, la incidencia social,

\section{Marco Referencial sobre Gestion de Riesgo en Colombia}

En Colombia el concepto de riesgo y su gestión ha tomado especial importancia por las afectaciones continuas de tipo natural a las cuales el territorio ha sido expuesto en los últimos años relacionados con inundaciones, deslizamientos y en general fenómenos asociados en su mayoría a las dinámicas ambientales de cambio climático; fenómenos de mayor incidencia en las zonas de pobreza, vulnerabilidad y menos desarrollo.

Tabla 1

Eventos en Colombia

\begin{tabular}{|c|l|}
\hline AÑO & \multicolumn{1}{|c|}{ EVENTO } \\
\hline 1906 & $\begin{array}{l}\text { Terremoto y Tsunami en la costa } \\
\text { pacífica sur }\end{array}$ \\
\hline 1973 & Incendio en la torre de Avianca \\
\hline 1979 & $\begin{array}{l}\text { Terremoto y Tsunami en la costa } \\
\text { pacífica sur }\end{array}$ \\
\hline $1982-1983$ & Fenómeno del Niño \\
\hline 1983 & Destrucción del 70\% de Popayán \\
\hline 1985 & Avalancha de Armero \\
\hline 1988 & Huracán Joan \\
\hline 1992 & Terremoto en Atrato Medio \\
\hline 1994 & Sismo y avalancha del rio Páez \\
\hline 1995 & Sismo de Pereira \\
\hline $1997-1998$ & Fenómeno del Niño \\
\hline 1999 & Terremoto Eje Cafetero \\
\hline 1999 & Fenómeno de la Niña \\
\hline $2006-2007$ & Fenómeno del Niño \\
\hline $2010-2011$ & $\begin{array}{l}\text { Fenómeno de la Niña } \\
\text { (mayor impacto) }\end{array}$ \\
\hline
\end{tabular}

Fuente: Los desastres en Colombia recuento histórico. http:// www.cra.gov.co/articulos. shtml?apc $=g D x x-1-\& x=3009$ 
El concepto de riesgo lo explica Soldano ${ }^{1}$ como: "La probabilidad de que ocurra algún hecho indeseable" o "La probabilidad de que una amenaza se convierta en desastre" y lo interrelaciona con factores culturales, históricos, políticos, socioeconómicos y ambientales. Gestionar el riesgo consiste en la capacidad para evitar que las amenazas se cumplan y pasen a ser desastres; lo cual se logra con criterios técnicos y operativos dedicados a la identificación de las amenazas.
En Colombia se ha conformado la estructura nacional y su marco normativo que establece los criterios mínimos para una eficaz gestión del riesgo, la Ley 46 de 1988 Crea el Sistema Nacional para la Prevención y Atención de Desastres -SNPAD-; el Decreto 93 de 1988 adopta el Plan Nacional. En el nivel regional y local se conforman los comités regionales y locales para la prevención y atención de desastres mediante la Ley 919 de 1989.

\section{Marco Normativo Nacional para la Gestión del Riesgo de Desastres}

\begin{tabular}{|c|c|}
\hline LEY/DECRETO & $\begin{array}{l}\text { DESCRICIÓN y OBLIGACIONES EN } \\
\text { TÉRMINOS DE INCORPORACIÓN DE LA } \\
\text { PREVENCIÓN Y REDUCCIÓN DE RIESGOS EN } \\
\text { LA PLANIFICACIÓN TERRITORIAL }\end{array}$ \\
\hline \multicolumn{2}{|c|}{ NORMA DE NORMAS } \\
\hline Constitución Política de Colombia & $\begin{array}{l}\text { Artículo 2: "...Las autoridades de la República es- } \\
\text { tán instituidas para proteger a todas las personas } \\
\text { residentes en Colombia, en su vida, honra, bienes, } \\
\text { creencias, y demás derechos y libertades, y para } \\
\text { asegurar el cumplimiento de los deberes sociales } \\
\text { del Estado y de los particulares..." }\end{array}$ \\
\hline \multicolumn{2}{|c|}{ CON RELACIÓN AL RIESGO } \\
\hline $\begin{array}{l}\text { Ley } 46 \text { de } 1988 \\
\text { Crea el SNPAD }\end{array}$ & $\begin{array}{l}\text { Crea el Sistema Nacional para la Prevención y At- } \\
\text { ención de Desastres y fija sus objetivos. }\end{array}$ \\
\hline $\begin{array}{l}\text { Decreto } 919 \text { de mayo } 1989 \\
\text { Organiza el SNPAD }\end{array}$ & $\begin{array}{l}\text { Obliga a las oficinas de Planeación a elaborar los } \\
\text { planes en armonía con las normas y planes sobre } \\
\text { prevención y atención de situaciones de desastre. } \\
\text { Obliga a las Corporaciones Autónomas Regionales } \\
\text { hacer inventarios y análisis de zonas de riesgos. } \\
\text { Obliga a todas las entidades territoriales destinar } \\
\text { recursos del presupuesto a la gestión del riesgo. } \\
\text { Incorpora automáticamente los planes de contin- } \\
\text { gencia y emergencia en los planes de desarrollo. }\end{array}$ \\
\hline
\end{tabular}


Cont..

\begin{tabular}{|c|c|}
\hline Decreto 93 de 1998 Adopta el PNPAD & $\begin{array}{l}\text { Orienta las acciones del Estado y de la sociedad } \\
\text { civil para la prevención, atención y reconstruc- } \\
\text { ción. } \\
\text { Determina todas las políticas, acciones y pro- } \\
\text { gramas, tanto de carácter sectorial como del or- } \\
\text { den nacional, regional y local. } \\
\text { Prioriza el conocimiento sobre riesgos de ori- } \\
\text { gen natural y antrópico y la incorporación de la } \\
\text { prevención y reducción de riesgos en la planifi- } \\
\text { cación. } \\
\text { Promueve la incorporación de criterios preventi- } \\
\text { vos y de seguridad en los Planes de Desarrollo. } \\
\text { Promueve la recuperación rápida de zonas af- } \\
\text { ectadas, evita duplicidad de funciones y dis- } \\
\text { minuye los tiempos en la formulación y ejecución } \\
\text { de proyectos. }\end{array}$ \\
\hline $\begin{array}{l}\text { CONPES } 3146 \text { de } 2001 \\
\text { Promueve la ejecución del PNAD }\end{array}$ & $\begin{array}{l}\text { Define las estrategias y recursos para la eje- } \\
\text { cución del Plan Nacional para la Prevención y } \\
\text { Atención de Desastres. }\end{array}$ \\
\hline CONPES 3318 del 2004 & $\begin{array}{l}\text { Autorización a la nación para contratar operacio- } \\
\text { nes de crédito externo con la banca multilateral } \\
\text { hasta por } \$ 260 \text { millones de dólares para finan- } \\
\text { ciar parcialmente el programa de reducción de } \\
\text { la vulnerabilidad fiscal del Estado frente a los } \\
\text { desastres naturales. }\end{array}$ \\
\hline $\begin{array}{l}\text { Resolución } 7550 \text { de } 1994 \\
\text { Prevención en Secretarias de Educación }\end{array}$ & $\begin{array}{l}\text { Obliga a las Secretarías de Educación a nivel De- } \\
\text { partamental y Municipal a incorporar la preven- } \\
\text { ción y atención de desastres dentro del Proyecto } \\
\text { Educativo Institucional, según el conocimiento } \\
\text { de las necesidades y riesgos de la región. }\end{array}$ \\
\hline Decreto 969 de 1995 & $\begin{array}{l}\text { Por el cual se organiza y reglamenta la Red Na- } \\
\text { cional de Centros de Reserva para la Atención } \\
\text { de Emergencias. }\end{array}$ \\
\hline Ley 322 de 1996 Crea el SNB & $\begin{array}{l}\text { Crea el Sistema Nacional de Bomberos. Se } \\
\text { reglamentó por la Resolución } 3580 \text { de } 2007\end{array}$ \\
\hline Ley 400 de 1997. & $\begin{array}{l}\text { Por el cual se adoptan normas sobre construc- } \\
\text { ciones sismos resistentes }\end{array}$ \\
\hline Directiva Ministerial N.12 de 2009 & $\begin{array}{l}\text { Prohíbe a las Secretarías de Educación de las } \\
\text { entidades territoriales interrumpir la prestación } \\
\text { del servicio educativo en situaciones de emer- } \\
\text { gencia. }\end{array}$ \\
\hline
\end{tabular}

Orienta las acciones del Estado y de la sociedad ción. den nacional, regional y local. prevención y reducción de riesgos en la planificación. Promueve la recuperación rápida de zonas afectadas, evita duplicidad de funciones y disminuye los tiempos en la formulación y ejecución de proyectos.

CONPES 3146 de 2001

Promueve la ejecución del PNAD

CONPES 3318 del 2004

Resolución 7550 de 1994

Prevención en Secretarias de Educación 
Cont.

\begin{tabular}{|c|c|}
\hline \multicolumn{2}{|c|}{ CON RELACIÓN AL DESARROLLO Y EL ORDENAMIENTO TERRITORIAL } \\
\hline $\begin{array}{l}\text { Ley } 9^{a} \text { de } 1989 \\
\text { Ley de Reforma Urbana }\end{array}$ & $\begin{array}{l}\text { Determina los parámetros de planificación y } \\
\text { gestión urbana en Colombia. } \\
\text { Obliga a incorporar en los Planes de Desarrollo as- } \\
\text { pectos de gestión del riesgo para la reubicación } \\
\text { de asentamientos en zonas de alto riesgo. }\end{array}$ \\
\hline Ley 02 de 1991 & $\begin{array}{l}\text { Por el cual se modifica la Ley } 9 \text { de } 1989 \text {. Entre } \\
\text { otras modifica el plazo para los inventarios de zo- } \\
\text { nas de alto riesgo. }\end{array}$ \\
\hline $\begin{array}{l}\text { Ley } 388 \text { de } 1997 \\
\text { Ley de ordenamiento territorial }\end{array}$ & $\begin{array}{l}\text { Obliga a la todos los municipios del país a formu- } \\
\text { lar planes de ordenamiento territorial teniendo en } \\
\text { cuenta la zonificación de amenazas y riesgos. } \\
\text { Obliga a todos los departamentos del país a pre- } \\
\text { star asistencia técnica para la formulación de los } \\
\text { planes de ordenamiento municipal. } \\
\text { Promueve el uso equitativo y racional del suelo, la } \\
\text { preservación y defensa del patrimonio ecológico } \\
\text { y cultural localizado en su ámbito territorial y la } \\
\text { prevención de desastres en asentamientos de } \\
\text { alto riesgo, así como la ejecución de acciones ur- } \\
\text { banísticas eficientes. }\end{array}$ \\
\hline Ley 02 de 1991 & Por el cual se modifica la Ley 9 de 1989 \\
\hline Ley 152 de 1994 & Ley Orgánica del Plan de Desarrollo. \\
\hline Decreto 879 DE 1998 & $\begin{array}{l}\text { Reglamenta las disposiciones referentes al orde- } \\
\text { namiento del territorio municipal y distrital y a los } \\
\text { planes de ordenamiento territorial. }\end{array}$ \\
\hline Decreto 2015 de 2001 & $\begin{array}{l}\text { Reglamenta la expedición de licencias de urbanis- } \\
\text { mo y construcción con posterioridad a la declara- } \\
\text { ción de situación de desastre o calamidad pública. }\end{array}$ \\
\hline \multicolumn{2}{|l|}{ Decreto 4002 de 2004} \\
\hline \multicolumn{2}{|c|}{ MEDIO AMBIENTE Y GESTIÓN DEL RIESGO } \\
\hline Ley 99 de 1993 Organiza el SINA & $\begin{array}{l}\text { Organiza el Sistema Nacional Ambiental (SINA) y } \\
\text { define su articulación con el SNPAD. } \\
\text { Define la prevención de desastres y las medidas } \\
\text { de mitigación como asunto de interés colectivo y } \\
\text { de obligatorio cumplimiento. } \\
\text { Obliga a las CAR a realizar actividades de análisis, } \\
\text { seguimiento, prevención y control de desastres, y } \\
\text { a asistir a las autoridades competentes en los as- } \\
\text { pectos en la prevención y atención de emergen- } \\
\text { cias y desastres. }\end{array}$ \\
\hline
\end{tabular}


Cont.

\begin{tabular}{|c|c|}
\hline DL 2811 de 1974 & $\begin{array}{l}\text { Código de los Recursos Naturales. Título II sobre } \\
\text { Protección Forestal (Art.241- } \\
242-243-244 \text { y 245) }\end{array}$ \\
\hline $\begin{array}{l}\text { Ley } 195 \text { de } 1994 \text { Aprueba el } \\
\text { Convenio de Diversidad Biológica }\end{array}$ & $\begin{array}{l}\text { - Obliga a inventariar y monitorear la biodivers- } \\
\text { idad } \\
\text { - Obliga al establecimiento de áreas protegidas } \\
\text { - Fomenta la rehabilitación y restauración de eco- } \\
\text { sistemas degradados en colaboración con la po- } \\
\text { blación local. } \\
\text { Promueve el respeto del conocimiento tradicional } \\
\text { e indígena sobre la biodiversidad }\end{array}$ \\
\hline CONPES 2834 de 1996 "Políticas de Bosques" & $\begin{array}{l}\text { Establece la necesidad de formular y poner en } \\
\text { marcha el "Programa Nacional para la Preven- } \\
\text { ción, Control y Extinción de Incendios Forestales } \\
\text { y rehabilitación de áreas afectadas }\end{array}$ \\
\hline Decreto 2340 de 1997. & $\begin{array}{l}\text { Por el cual se dictan unas medidas para la orga- } \\
\text { nización en materia de prevención y mitigación de } \\
\text { incendios forestales y se dictan otras disposicio- } \\
\text { nes. }\end{array}$ \\
\hline CONPES 2948 de 1997 & $\begin{array}{l}\text { Recomendó acciones para prevenir y mitigar los } \\
\text { posibles efectos del Fenómeno del Niño } 1997 .\end{array}$ \\
\hline $\begin{array}{l}\text { Primera Comur } \\
\text { ante CMNUCC }\end{array}$ & $\begin{array}{l}\text { Elabora el primer inventario nacional de Gases de } \\
\text { Efecto Invernadero. } \\
\text { Identifica los ecosistemas más susceptibles al } \\
\text { cambio climático } \\
\text { Plantearon las primeras medidas de adaptación } \\
\text { para el país. }\end{array}$ \\
\hline $\begin{array}{l}\text { Lineamientos de Política de } \\
\text { Cambio Climático } 2002\end{array}$ & $\begin{array}{l}\text { Mejora la capacidad de adaptación a los impac- } \\
\text { tos del cambio climático } \\
\text { Promueve la reducción de emisiones por fuente y } \\
\text { absorción por sumideros de GEl } \\
\text { Promueve la investigación, divulgación y concien- } \\
\text { tización pública } \\
\text { Fortalecer el sistema de información en Cambio } \\
\text { Climático } \\
\text { Desarrollar mecanismos financieros }\end{array}$ \\
\hline CONPES 3242 de 2003 & $\begin{array}{l}\text { Establece y reglamenta la venta de Servicios Am- } \\
\text { bientales de Mitigación de Cambio Climático }\end{array}$ \\
\hline
\end{tabular}


Cont.

\begin{tabular}{|l|l|}
\hline \multirow{5}{*}{ Decreto 350 de 1999} & $\begin{array}{l}\text { Dicta disposiciones para hacer frente a la emer- } \\
\text { gencia económica, social y ecológica causada por } \\
\text { el terremoto ocurrido el } 25 \text { de enero de 1999. Las } \\
\text { Corporaciones } \\
\text { Autónomas Regionales con jurisdicción en la zona } \\
\text { de desastre apoyarán y asistirán técnicamente a } \\
\text { los municipios afectados en el área de su jurisdic- } \\
\text { ción, en la incorporación de los determinantes y cri- } \\
\text { terios ambientales en sus planes de ordenamiento }\end{array}$ \\
\hline \multirow{2}{*}{$\begin{array}{l}\text { Segunda Comunicación Nacional ante CMNUCC } \\
2010\end{array}$} & $\begin{array}{l}\text { Presenta el inventario nacional de fuentes y sum- } \\
\text { ideros de gases de efecto invernadero 2000 y } \\
\text { 2004 } \\
\text { Identifica oportunidades de reducción y captura } \\
\text { de gases efecto invernadero } \\
\text { Determina la alta vulnerabilidad de Colombia ante } \\
\text { los efectos adversos del cambio climático } \\
\text { Expones acciones que se han adelantado en ma- } \\
\text { teria de adaptación } \\
\text { Determina prioridades de acción }\end{array}$ \\
\hline Ley 1523 del 2012 & $\begin{array}{l}\text { Adopta la política nacional de gestión del riesgo } \\
\text { de desastres y se establece el sistema nacional de } \\
\text { gestión del riesgo de desastres y se dictan otras } \\
\text { disposiciones. }\end{array}$ \\
\hline Política Nacional de Biodiversidad & $\begin{array}{l}\text { Sus objetivos son: conservar, conocer y utilizar la } \\
\text { biodiversidad. } \\
\text { Hace énfasis en la distribución justa y equitativa } \\
\text { de los beneficios derivados de la utilización de la } \\
\text { biodiversidad. }\end{array}$ \\
\hline
\end{tabular}

Fuente: Plan Departamental de Gestión de Riesgo del Atlántico.

Serie Ambiente y Ordenamiento Territorial: guía metodológica 1 - Incorporación de la prevención y la reducción de riesgos en los procesos de Ordenamiento Territorial.

El plan nacional para la prevención y atención de desastres ${ }^{2}$ se estructura alrededor de 4 estrategias:

2 Dirección Nacional de Planeación http://www.dnp.gov co/Programas/Ambiente/Gesti\%C3\%B3ndelRiesgo/ MarcoLegal.aspx a. El conocimiento sobre riesgos de origen natural y antrópico.

b. La incorporación de la prevención y reducción de riesgos en la planificación.

c. El fortalecimiento del desarrollo institucional, y

d. La socialización de la prevención y la mitigación de desastres. 
Para la financiación del funcionamiento de la estructura desde el nivel nacional se crea el Fondo Nacional de Calamidades mediante el Decreto 1547 de 1984 el cual servirá como "apoyo económico indispensable para las labores de prevención, atención y recuperación, y en caso de situaciones de desastre y calamidad, administrará los aportes en dinero, y supervisará el manejo y control del inventario de los centros de reserva para emergencias"3.

Podemos asegurar que el desarrollo normativo en Colombia para el manejo del riesgo y la prevención ha sido un proceso de construcción desde las instancias nacionales, tratando en una estructura organizacional muy densa de llevar su aplicabilidad a la escala local; propósito que aún es en ciudades pequeñas algo efímero por muchos aspectos:

- La extensión del marco normativo no permite su clara articulación y la aplicabilidad eficiente en caso de desastres y mucho menos para activar los mecanismos preventivos.

- La estructura del sistema al tratar de colocar en interlocución tantos actores se dilata en las escalas de aplicabilidad, evitando decantar el proceso a los niveles locales en un territorio tan extenso; situación que se dificulta con el hecho de que se elaboraron los planes municipales de gestión de

${ }^{3}$ Dirección Nacional de Planeación http://www.dgpad.gov.co/ entidad/normatividad.htm riesgo sin existir como referente de política un Plan articulador a nivel nacional que solo recientemente se empieza a construir.

- El hecho de que el Fondo de calamidades dependa del comité nacional convierte el modelo de inversión dentro de un esquema tan complejo en una dinámica donde los recursos en la mayoría de los casos terminan disgregados en el encadenamiento del sistema y no permite su ejecución inmediata en los momentos de mayor "urgencia".

- La falta de claridad desde la norma sobre la procedencia de los recursos para el fortalecimiento institucional de las instancias que se asocian al manejo de la prevención del riesgo.

- Finalmente, en términos de articular la norma y los procesos de riesgo con el Ordenamiento territorial y la planeación no es muy convincente pensar que este proceso se cumple solamente con la incorporación a los POT de los planos de riesgo de las áreas territoriales; sobre todo teniendo en cuenta que en muchas ciudades los POT fueron configurados desde el año 2000 sin seguimiento a estos temas y actualmente en su reformulación fue mucho lo que se desarrolló en 13 años o nada lo que se cumplió sin haber seguimiento y control de parte de las instancias nacionales quienes tienen competencia para garantizar su ejecución. 


\section{La cooperación internacional en el tema de gestión de riesgo}

Los últimos acontecimientos de desastres naturales en Colombia y a nivel mundial son el reflejo de los cambios y trastornos causados al ecosistema natural en el que vivimos: el efecto invernadero, el cambio climático, terremotos, tsunamis y el déficit de los recursos energéticos y alimenticios dan cuenta de ello.

La posibilidad de prevenir estos eventos es la apuesta en materia de políticas nacionales e internacionales de cooperación internacional, marco en el cual la gestión del riesgo de desastres para ser efectiva y viable debe conllevar a una mayor integración del enfoque conceptual del desarrollo sostenible teniendo en cuenta como asegura Vadala, S (2012):

- No es posible esperar un alto nivel de desarrollo para luego incluir formas de prevención/mitigación.

- La asistencia de la cooperación internacional a los damnificados es más costosa que la prevención/mitigación.

- Los países más desarrollados también necesitan una revisión continua de la vulnerabilidad.

- La correcta integración del enfoque de gestión del riesgo de desastres representa "bienes públicos" internacionales y, en particular, tanto el impacto de los desastres como la res- puesta a ellos tiene cada vez mayor carácter transnacional/multinacional ${ }^{4}$.

La cooperación internacional ha creado para atender los asuntos de la Gestión del riesgo en el mundo y en especial en América Latina distintos organismos con diferenciación de funciones en el acompañamiento técnico y el financiamiento de acciones para la construcción y fortalecimiento de los entes locales y los sistemas de atención de riesgo y de desastres. Para Colombia se ha diseñado la Estrategia Nacional de Cooperación Internacional a implementarse entre el 2012 y el $2014^{5}$.

La estrategia articulada al plan nacional de desarrollo actual, busca a través de la Agencia Presidencial de Cooperación Internacional fortalecer varios aspectos:

a. Empleo, pobreza, seguridad y transversalmente los temas de buen gobierno, competitividad, sostenibilidad ambiental y gestión del riesgo como país vulnerable al cambio climático.

b. Política y gestión unificada de la cooperación internacional, mediante la articulación de todos los sectores y entes territoriales.

\footnotetext{
${ }^{4}$ Vadala, Sebastián. (Prologo) Gestión Integrada del Riesgo de Desastres. ELACID, 2011.

${ }^{5}$ http://www.minminas.gov.co/minminas/downloads/UserFiles/ File/Planeacion/Cooperacion\%20Internacional/ENCI\%20 2011-2014.pdf
} 
c. Diversificación de las fuentes de cooperación.

d. Alcanzar las metas de gestión y financiación.

e. Poder manejar los recursos de cooperación a través del presupuesto general de la nación, con eficacia y transparencia

f. Lograr la equidad en la distribución territorial de los recursos de cooperación.

g. Consolidación de la oferta de cooperación sur - sur.

En el marco de la estrategia de cooperación una de las áreas de interés identificadas para la inversión internacional es la necesidad de fortalecer el sistema nacional de gestión de riesgos y desastres, articuladamente con la formulación de la política nacional para cuyo objetivo están definidos los siguientes lineamientos prioritarios:

a. Desarrollo e implementación de la política. Proceso que apenas inicia.

b. Incorporación de la gestión del riesgo en los POT, POMCA, PGAR. Proceso que se encuentra en estado de inicio a partir de las actualizaciones de los POT, dependiendo su avance de la inversión municipal y la cohesión institucional local y regio- c. Fortalecimiento de los sistemas nacionales y regionales de información para la gestión del riesgo. No hay inversión municipal para esta destinación en la mayoría de los casos.

d. Fortalecimiento de las redes para la atención de emergencias. Son activadas después de la emergencia.

e. Restablecimiento de las víctimas y de los territorios afectados. Proceso que tiene una duración de hasta dos y tres años.

Otra área de acción serán las de mitigación y adaptación al cambio climático, cuyos objetivos estarán encaminados a la producción ambientalmente sana y sostenible y el cumplimiento de Colombia de los objetivos de la Convención de Naciones Unidas sobre cambio climático. Actualmente el país se encuentra formulando la política para el desarrollo sustentable por parte de la Dirección Nacional de Planeación y Ministerio de vivienda y desarrollo urbano.

\section{El Desarrollo Integral y Sostenible Asociado a la Gestión del Riesgo}

El modelo de desarrollo sostenible debe ser la combinación de un grado de desarrollo económico y de calidad de vida que no comprometa la capacidad del medioambiente para sustentar a la población del futuro, proporcionando un grado de bienestar económico a las genera- 
ciones actuales y venideras a la vez que se mantiene el buen estado del medioambiente ${ }^{6}$.

Garantizar la efectividad del modelo incluye plantear políticas locales y estrategias de financiación para asegurar el control demográfico, el uso eficiente de los recursos naturales, la reducción de residuos y contaminación, las situaciones de provecho para todos, la definición de los límites medioambientales, la depuración del concepto de economía de mercado, formación y cambios sociales y culturales; todos a su vez, factores de riesgo y vulnerabilidad presentes en los territorios.

Se entiende como desarrollo, la condición de vida de una sociedad en la cual las necesidades auténticas de los grupos y/o individuos se satisfacen mediante la utilización racional, es decir sostenida de los recursos y los sistemas naturales. Para ello se utilizarían tecnologías que no se encuentran en contradicción con los elementos culturales de los grupos involucrados.

Este concepto integra elementos económicos, tecnológicos, de conservación y utilización ecológica, así como lo social y político. La esfera de poder, dentro del contexto social se hace necesaria como forma organizativa y de cohesión legítima, legal y funcional dentro de grupos sociales

6 Informe Bruntland "Comisión Mundial sobre y como instancia de toma de decisiones entre individuos?.

El concepto de desarrollo trasciende el mero ámbito económico y tiene claras connotaciones políticas y sociales. La modernización económica es sólo un aspecto de la modernización de una sociedad. Politólogos y sociólogos vienen trabajando desde los años cincuenta en las dimensiones políticas y sociales del desarrollo y que merecen una atención especial.

La misma economía ha reconocido recientemente el papel de las instituciones en el crecimiento económico y en los procesos de desarrollo. Además, el reciente énfasis en conceptos como el de desarrollo humano otorga una mayor importancia al entorno político e institucional. Dentro de las instituciones, la cultura en su sentido antropológico, empieza a ser abordada como un elemento más del análisis del desarrollo. La modernización entraña cambios económicos, pero también políticos, sociales y culturales ${ }^{8}$.

El enfoque del desarrollo integral debe definir e incluir todas las dimensiones:

La dimensión económica, referida a la capacidad local para organizar los factores de producción con niveles de producti-

${ }^{7}$ Diccionario de Economía Zona Económica. http://www. zonaeconomica.com/concepto-desarrollo

${ }^{8}$ Gonzalo Escribano, Teorías del Desarrollo Económico p.10 
vidad suficientes para ser competitivos en los mercados y una fuerte integración entre las instituciones y la economía local favorable a la innovación, a la adaptación y a las siempre cambiantes condiciones de la realidad productivas ${ }^{9}$.

\section{La dimensión política e institucional,} las iniciativas locales crea un entorno local favorable a la producción e impulsan el desarrollo sostenible ${ }^{10}$.

La dimensión humana, todos los actos humanos requieren una valoración previa. Esta valoración se realiza en atención a unos valores, los cuales, constituyen la esfera de la moral. Y como ésta afecta poderosamente las elecciones y decisiones de las personas, ejercen una gran influencia en todos los campos de la vida humana. La ética afecta las valoraciones en cuanto a ¿qué y cómo producir? , los procesos empresariales y en última instancia, define un tipo de convivencia en sociedad. De esta forma, la moral se desempeña trascendentalmente en el Desarrollo Endógeno, pues, determina el marco valorativo social-individual desde donde se propone el futuro y se preparan las condiciones necesarias para su consecución definitiva.

La dimensión ambiental, El componente ambiental determina la preferencia por los recursos locales y regionales. El ecodesarrollo es una clase de desarrollo que insiste en buscar soluciones específicas a los problemas particulares de cada región ecológica, teniendo en cuenta la información ecológica y sociocultural reduciendo al mínimo de daños al ambiente y realizando mejor uso de los subproductos.

La dimensión socio cultural, manifiesta el hecho de que los valores e instituciones locales sirven de base al proceso de desarrollo y se fortalecen en el ejercicio de la propia estrategia, constituye un conjunto de elementos entre los que podemos resaltar: una profunda identidad local proyectada en una cultura propia; la consideración de la capacidad empresarial y de iniciativa como valor social positivo en la colectividad; la existencia de estructuras familiares con vínculos intergeneracionales sólidamente cohesionadas y que hacen de ella una unidad de renta y producción; una organización social con un nivel suficiente de actividades mercantiles; y un sistema urbano desarrollado sobre el área donde el sistema industrial se articula ${ }^{11}$.

El fenómeno del Desarrollo Endógeno impone como elemento clave de sustentación, la existencia de organizaciones eficientes que se dediquen a la producción de bienes y servicios. Además, se requiere la inclusión de otras organiza-

${ }^{11}$ Peña Sánchez Antonio Rafael. Pág. 61 y 62. 
ciones a los fines, de dar apalancamiento al sistema productivo general y a la comunidad social interesada en el Desarrollo Endógeno.

La integralidad de todas las dimensiones de análisis para estimar las condiciones de desarrollo de las comunidades deberá ser una metodología a desarrollar y aplicar con mayor profundidad y asi, en la medida en que se fortalezcan las organizaciones en cada una de las dimensiones podremos evaluar en el tiempo y con base en indicadores de base los cambios en las estructuras locales; alcanzando las metas hacia un desarrollo óptimo e incluyente.

Es fundamental emprender bajo un enfoque participativo el diagnostico de los escenarios para cada una de las dimensiones, identificando los factores de riesgo y vulnerabilidad presentes, lo cual nos dará premisas para una planeación por escenarios en la identificación de los riesgos, lo que permitirá crear las herramientas para la prevención y mitigación.

Amenazas naturales: biológicas, hidrometeorológicas, geológicas.

Amenazas antrópicas: degradación ambiental, amenazas tecnológicas, geopolíticas y socio-económicas ${ }^{12}$.

12 Gabriel Orozco Restrepo y Oscar Guevara Arévalo "Términos principales relativos a la reducción del riesgo de desastres" de la Organización de Naciones Unidas (2004) y en el Informe del Foro Económico Mundial "Riesgos Globales 2011". ELACID-USBCTG, 2012.

\section{Un Modelo de Gestion de Riesgo con Enfoque de Derechos}

Se define derechos humanos como: "un conjunto de facultades e instituciones que, en cada momento histórico, concretan las exigencias de la dignidad, la libertad y la igualdad humanas, las cuales deben ser reconocidas positivamente por los ordenamientos jurídicos a nivel nacional e internacional"13

La importancia del concepto y de describirlos radica en poder garantizar su cumplimiento para toda la humanidad, apelando a que solo así se mitigaran las desigualdades en los territorios y se garantizará una inserción absoluta y equilibrada en cualquier modelo de desarrollo que se implemente.

Los gobiernos locales como administradores de los entes territoriales adoptan una dimensión funcional por cuanto su acción específica se reduce a aplicar lo que la ley le define, esta postura de los gobiernos no es suficiente para garantizar el buen desempeño de la institucionalidad; debiendo estar asociado a una gerencia pública que recoja los requerimientos y necesidades de los sectores no gubernamentales y sociales, para que de manera conjunta se generen sinergias que permitan hacer una optimización de los recursos

\footnotetext{
13 Pérez, Luño. Definición de derechos humanos. http://plesiologos.blogspot.com/2008/02/definicinde-los-derechos-humanos-segn.html . Comunidad de investigación filosófica.
} 
disponibles y un logro de los resultados en el menor tiempo.

La Constitución Política de 1991, establece que Colombia es un estado social de derecho y contempla dentro de sus fines protección de los bienes, la vida y la honra de los colombianos, otorgándoles competencias a los entes locales para hacer cumplir los derechos de sus ciudadanos. Adicionalmente, la CP estipula los principios de corresponsabilidad para incorporar a las organizaciones sociales como parte fundamental del desarrollo local.

Las entidades y en general todas las estructuras políticas y administrativas en Colombia bajo el mandato constitucional en mención, deben asumir como parte de sus funciones el fortalecimiento de las relaciones intersectoriales desde el nivel local al nacional y en el manejo del "riesgo" brindar todas las garantías para la salvaguarda de los derechos de sus conciudadanos algunas entidades para dar estas garantías aseguran en buena parte recursos para la Identificación de las organizaciones sociales, apoyo para la estructuración operativa de estas organizaciones, acompañamiento en la gestión local y fortalecimiento de la participación interinstitucional, acompañamiento técnico para la definición de políticas y planes asociados.

Cabe anotar que en un contexto amplio tales inversiones no se reflejan en los avances por el respeto de los derechos, y tampoco en los niveles de gobernabilidad de los administradores locales, creando desconfianza entre las organizaciones sociales por este tipo de procesos situación que se refleja en la falta de inclusión, la poca participación, el enfoque asistencialista y la falta de seguimiento sobre los resultados.

La educación como derecho fundamental debe el estado garantizarla, entendiendo que abarca todos los escenarios y niveles posibles de interacción que permita mejorar la convivencia ciudadana, las relaciones sociales y el manejo de un ambiente sostenible; lo cual con la participación de todos ayudara a la construcción de una visión de ciudad y de región enmarcada en un modelo sostenible de desarrollo.

Bajo este precepto el eje de educación y cultura será un elemento fundamental que nos permita en el territorio encauzar una estrategia de formación y de identificación de factores culturales y elementos claves sobre el comportamiento del ser frente la ocupación y uso del territorio.

Desde la formación se podrá llevar a todas las organizaciones y representaciones sociales el conocimiento de sus derechos fundamentales y los temas relevantes para el fortalecimiento institucional de manera transversal con la gestión del riesgo y el enfoque de derechos. Será primordial transferir conocimiento y capacidades a las comunidades en temas de derechos, inclusión, género, las dinámicas interculturales, los instrumentos de parti- 
ción, la identificación y reconocimiento de contextos históricos, geográficos, políticos y sociales, herramientas e instrumentos para la gestión y la articulación intersectorial en todos los niveles.

El conocimiento de las características culturales de cada territorio a intervenir será información esencial para determinar estrategias sobre los modos de interacción con cada territorio y sus formas de representatividad social.

Bajo el concepto de administrar los riesgos como un derecho de los habitantes sobre el cual la institucionalidad debe dar garantías la ley 9 de 1989, determino la necesidad de incorporar a los planes de desarrollo acciones claras para la intervención territorial, y en el mismo año el decreto ley 919 ordena a los entes territoriales la inclusión de la prevención del riesgo en los planes de desarrollo sectorial y en todo proceso de planificación.

La ley 388 de 1997 es más explícita al requerir incorporar a la construcción de los planes de ordenamiento "políticas, directrices y regulaciones sobre prevención de amenazas y riesgos naturales, el señalamiento y localización de las áreas de riesgo para asentamientos humanos, así como las estrategias de manejo de zonas expuestas a amenazas y riesgos naturales"14.

\footnotetext{
14 Serie Ambiente y Ordenamiento Territorial: Guía metodológica para la incorporación de la prevención y la reducción de riesgos en los procesos de Ordenamiento Territorial. MAVDT, 2005
}

Avanzar en este camino implica entender el desarrollo territorial desde un enfoque en el cual se identifiquen todos los escenarios existentes y los posibles, para gestar un modelo de planeación que articule las dinámicas sociales, ambientales y económicas al ordenamiento físico; acompañado de un marco de formulación de políticas públicas que favorezcan la intervención de los municipios y su ordenamiento con unas bases sólidas de inversión, seguridad y gobernabilidad.

Sin duda los procesos de ordenación del espacio físico de los territorios no debe ser otro que aquel que pueda responder a las dinámicas sociales y económicas y para ello es de suma importancia la identificación de todos los escenarios de riesgo existentes, pero también la gestión en recursos para llevar a la escala mínima territorial, la participación que se requiere de todas las organizaciones de base, públicas y privadas en el reconocimiento de los escenarios, la aplicabilidad de las normas, la actualización de los sistemas de información y en general la efectividad en la minimización de los riesgos identificados.

\section{Conclusiones}

Avanzar en este camino implica entender el desarrollo de los municipios desde un enfoque en el cual se identifiquen todos los escenarios existentes y los posibles, para gestar un modelo de planeación que articule las dinámicas sociales, ambien- 
tales y económicas al ordenamiento físico ideal de cada territorio.

Debe estructurarse un marco de políticas públicas que favorezcan la intervención de los municipios y su ordenamiento con unas bases sólidas de inversión, seguridad y gobernabilidad. El aporte para encausar estas dinámicas actuales frente al tema se resume en tres estrategias de base y que al parecer son fundamentales desarrollar en el marco del modelo y son:

\section{a. Fortalecimiento institucional y orga-}

nizacional: el planteamiento actual en el tema de fortalecimiento radica en estimar que el nivel de desarrollo de un territorio está ligado a la capacidad local de la sociedad civil para la inclusión en los temas de política y gobernabilidad, proceso que resulta ser positivo y altamente beneficioso en la medida que tales comunidades poseen el conocimiento y manejo de los escenarios de participación y las herramientas técnicas, conceptuales y metodológicas para gestar una transformación; el contrario de esto el fracaso del desarrollo económico y social radica en las debilidades existentes en sus sistemas institucionales.

Haciendo uso de las tipologías de intervención establecidas por la cooperación técnica ${ }^{15}$, podríamos proponer

\footnotetext{
15 Luis Cámara López, Cooperación técnica para el fortalecimiento institucional. CIDEAL. http:// fortalecimiento.cideal.org/sitefiles/adjuntos/4d6cdec77a
} de83f4ceeed1f63c389ec2.pdf los siguientes sectores de actuación y líneas de intervención, para ser aplicados en el tema que se desarrolla:

- Fomento de la participación social y la democracia participativa.

Líneas de intervención: Fortalecimiento de organizaciones de la sociedad civil, Defensa de derechos y libertades fundamentales.

- Promoción de un entorno propicio para el desarrollo económico y social.

Líneas de intervención: Concertación y diálogo social, Fortalecimiento de las administraciones fiscal, de ingreso y gasto público, Apoyo a procesos de integración regional.

- Administración pública, buen gobierno y procesos de descentralización.

Líneas de intervención: Gestión de políticas públicas, Descentralización y fortalecimiento de administraciones regionales y locales, Transparencia y lucha contra la corrupción.

El ejercicio debe ser aplicado al nivel de las organizaciones, entendido como las de la sociedad civil, institucionales y/o las productivas entre otras.

Para el enfoque de trabajo cuyo objetivo será el fortalecimiento de las organizaciones sociales y de base o productivas frente al conocimiento, manejo e intervención en temas de gestión de riesgo la estrategia se acoge a los modelos 
de análisis de los entes que se vinculan donde hay que entender que las organizaciones poseen un comportamiento interno -organizacional- y un manejo de las relaciones y dependencias con el entorno donde se vinculan.

Bajo esta perspectiva habrá que proponer una intervención de las organizaciones en cada territorio donde se aborden tres aspectos fundamentales: su estructura organizativa y funcional, su capacidad técnica y operativa y su capacidad de gestión y relacionamiento.

\section{b. Refuerzo de capacidades en el terri-}

torio: Se podrá definir como el cumulo de conocimientos, herramientas y recursos físicos y sociales que posee un territorio para crear escenarios de participación, gestión, incidencia, mitigación de riesgos y/o respuestas inmediatas a situaciones de riesgo y vulnerabilidad. En la medida de que se logre tal fortalecimiento cada territorio podrá ser más competitivo y menos vulnerable al riesgo tanto natural como antrópico.

ONU-HABITAT ${ }^{16}$ establece varios objetivos que pueden ser cumplidos con el logro del fortalecimiento de las capacidades territoriales, relacionados con: la capacidad de los gobiernos locales para la

${ }^{16}$ ONU - HABITAT: Por un mejor futuro urbano. Oficina Regional para América Latina y el Caribe. http://www. onuhabitat.org/index.php?option=com_content\&view=ar ticle\&id $=66 \&$ Itemid $=33$ prevención y la reducción de desastres en los asentamientos humanos, la promoción de políticas y estrategias para fortalecer la gestión urbana y manejo del riesgo en los asentamientos humanos, fortalecer la cooperación e intercambio regional en la promoción de actividades orientadas a reducir el riesgo en las ciudades, promoción y fortalecimiento de la sociedad civil en la gestión local del riesgo como actores del desarrollo local.

\section{c. Información y comunicación:} Lograr obtener información veraz para el conocimiento y manejo del territorio con la finalidad de responder oportunamente a la mitigación y en el mejor de los casos evitar el riesgo, es la premisa que debe sostener el esquema de fortalecimiento institucional y organizacional, aplicado en cualquier territorio.

En este aspecto el deseo del fortalecimiento organizacional está enfocado hacia los siguientes objetivos:

- Diagnóstico de las situaciones de riesgo.

- Actualización permanente del estado de vulnerabilidad del territorio.

- Conocimiento en tiempo real de los cambios y modificaciones territoriales.

- Herramientas e instrumentos técnicos y tecnológicos para la lectura de la información y su actualización permanente. 
- Personal humano capacitado para la lectura de la información y manejo de esta para la mitigación o respuesta inmediata en caso de desastre.

- Capacidad social e institucional para aplicar mecanismos de recuperación y contingencia.

- Fortalecer y facilitar el acceso a la información, documentación y sistematización de experiencias a todos los actores claves de la gestión local del riesgo y de la gestión urbana, debe ser el cometido.

\section{Referencias}

PNGR, Gobierno de Costa Rica. http:// www.cne.go.cr/Documentos/planificacion/plan_nacional_para_gestion_ riesgo_2010_2015.pdf

Ministerio del Interior (2012). Ley Sistema Nacional de Gestión del Riesgo. Por la cual se adopta la política nacional de gestión del riesgo de desastres y se establece el sistema nacional de gestión del riesgo de desastres y se dictan otras disposiciones. Imprenta Nacional de Colombia. [Revisión diciembre del 2012]. Disponible en: http://www.mij.gov.co/Ministerio/ Library/News/Files/CARTILLA\%20 SISTEMA\% 20 NACIONAL\% 20 DE \% 20 GESTI\%C 3\% 93 N\% 20 DEL\%20RIESGO112.PDF
Gobierno de Costa Rica (2010-2015) Plan Nacional para la Gestión del Riesgo. Marco estratégico para la aplicación de la política de gestión del riesgo. Comisión Nacional de Prevención de Riesgos - Enlace de Esfuerzos. Departamento de Planificación. [Revisión diciembre del 2012]. Disponible en: http://www. cne.go.cr/Documentos/planificacion/plan_nacional_para_gestion_ riesgo_2010_2015.pdf

Informe de la Conferencia Mundial sobre la reducción de los desastres. Naciones Unidas (2005). A/ conf206/6. GE 05_61032 (s) 180405 - 200405. [Revisión diciembre del 2012]. Disponible en: www.unisdr. org/files/1037_finalreportwcdrspanish1.pdf

Estrategia Internacional para la reducción de desastres (2012 Dic) Las Américas ONU. Boletín para América Latina y el Caribe No. 14, 99. 1999. Disponible en: www.eird. org/esp/revista/No.14_99/editori2. htm\#sec4

Soldano, A. (2009) Conceptos sobre Riesgo. Síntesis temática realizada para el Foro Virtual de la RIMD (2012) Capacitación en Teledetección Aplicada a la Reducción delRiesgo por Inundaciones. CONAE Y OEA, Argentina. Disponible en: http://www.rimd.org/advf/documento s/4921a2bfbe57f2.37678682.pdf 
Moreno, J. (2011) Gestión de riesgo en el sector de agua potable y saneamiento básico. Comisión de Regulación de Agua Potable y Saneamiento Básico. Taller sobre gestión integral del riesgo y la regulación económica de los servicios públicos de acueducto y alcantarillado. CRA MAVDT - BID.

Gobernación del Atlántico (2012) Plan Departamental de Gestión de Riesgo del Atlántico. Proyecto "fortalecimiento de las capacidades institucionales para la implementación de prácticas locales de gestión del riesgo como medida de adaptación al cambio climático en la zona insular y costera del Caribe Colombiano" COL/72959 PNUD-UNGRD, Unión Europea.

Serie Ambiente y Ordenamiento Territorial (2005) Guía metodológica 1 - Incorporación de la prevención y la reducción de riesgos en los procesos de Ordenamiento Territorial. Ministerio de Ambiente, Vivienda y Desarrollo Territorial - Dirección de Desarrollo Territorial. República de Colombia.

Departamento Nacional de Planeación DNP. Marco Legal (2012). Disponible en: http://www.dnp. gov.co/Programas/Ambiente/ Gesti\%C3\%B3ndelRiesgo/MarcoLegal.aspx
Departamento Nacional de Planeación DNP. Marco Legal (2012). Disponible en: http://www.dgpad.gov.co/entidad/ normatividad.htm

Orozco, R.; Guevara A. (2011). Gestión Integrada del Riesgo de Desastres. Vadala, Sebastián (Prólogo) Cuadernos de Cooperación para el Desarrollo N. ${ }^{\circ}$ 4. Escuela Latinoamericana de Cooperación y Desarrollo.

Estrategia Nacional de Cooperación Internacional de Colombia, (2012 - 2014). Pdf Disponible en: http://www.minminas. gov.co/minminas/downloads/UserFiles/ File/Planeacion/Cooperacion\%20Internacional/ENCl\%202011-2014.pdf

Comisión Mundial Para el Medio Ambiente y el Desarrollo. Informe Brundtland (1987). ONU [Revisión diciembre del 2012]. Disponible en: http://ringofpeace.org/environment/brundtland. html www.un-documents.net/wced-ocf. $\mathrm{htm}$

Economías Regionales (2012 Dic) Clase $\mathrm{N}^{\circ} 14$ - Sobre el concepto de desarrollo (regional y/o local). Disponible en: http://chames-ecoregionales.blogspot. com/2008/08/clase-n14-sobre-elconcepto-de.html

Meza, C; Domenech, A. (2011). Módulo de trabajo medio ambiente urbano y desarrollo territorial. Especialización en medio ambiente urbano y desarrollo territorial. Universidad de San Buenaventura, Cartagena. 
Peña, S. (2004). Las disparidades económicas intrarregionales en Andalucía. Servicio de Publicaciones de la Universidad de Cádiz. ISBN: 978-84-9828-084-5.

Reyes, G (2012). Comercio y Desarrollo: Bases Conceptuales y Enfoque para América Latina y el Caribe Disponible en: http://www.zonaeconomica.com/concepto-desarrollo.

Escribano, G (2012 Dic) Teorías del desarrollo económico. Disponible en: http://www.uned.es/deahe/ doctorado/gescribano/teorias\%20 desarrollo\%20oei.pdf
Pérez, L. (2012) Definición de derechos humanos. Disponible en: http:// plesiologos.blogspot.com/2008/02/ definicin-de-los-derechos-humanossegn.html - Comunidad de investigación filosófica.

Cámara, L. (2012 Dic) Cooperación técnica para el fortalecimiento institucional. CIDEAL. Disponible en: http://fortalecimiento.cideal.org/sitefiles/adjuntos/4 d6cdec77ade83f4ceeed1f63c389ec2. pdf

ONU - HABITAT. (2012) Por un mejor futuro urbano. Oficina Regional para América Latina y el Caribe. Disponible en: http://www.onuhabitat.org/index. php?option=com_content\&view=articl e\&id=66\&ltemid=33. 